\title{
Clinical Observation of 29 Cases of Tonifying Kidney and Spleen, Detoxification of Throat Traditional Chinese Medicine in the Treatment of IgA Nephropathy \\ Di ZOU ${ }^{1, a}$, Hong-an WANG ${ }^{2}$, Yin-ping $W_{A N G}{ }^{3}$ and Shou-lin ZHANG ${ }^{4 b^{*}}$
}

${ }^{1,2,3,4}$ Department of Nephrology, the First Affiliated Hospital to Changchun University of Chinese Medicine, Changchun, Jilin province, China,130021.

aaggiezou@163.com , bshoulin-z@163.com

Keywords: $\lg$ A nephropathy, Tonifying kidney and spleen, Detoxification of throat, $24 \mathrm{~h}$ urine protein.

\begin{abstract}
Purpose:To observe the clinical effect of tonifying kidney and spleen, detoxification of throat traditional Chinese medicine in the treatment of $\operatorname{IgA}$ nephropathy. Methods:60 patients with IgA nephropathy were randomly divided into treatment group and control group with 30 cases in each. The treatment group give tonifying kidney and spleen, detoxification of throat of Chinese herbal medicine, daily 1 agent. The control group was treated with Lotensin $10 \mathrm{mg}$, once daily orally. The course of treatment in the two groups are three months. Compared with the two groups before and after treatment, TCM syndrome score, $24 \mathrm{~h}$ urinary protein, urinary red blood cell count and adverse reaction. Results: In the treatment group, 1 cases loss. In the control group, 3 cases loss. Treatment group of TCM syndrome lumbar pain, sore throat, fatigue, edema, low sodium, xerostomia score decreased after treatment $(P<0.05)$. Compared with before and after treatment, the treatment group with $24 \mathrm{~h}$ urinary protein and urinary red blood cell count were significantly reduced $(P<0.01)$.Control group after only $24 \mathrm{~h}$ urinary protein decreased significantly $(P<0.05)$, the difference in urinary red blood cell count no statistical significance $(P>0.05)$. The two groups after treatment, the treatment group $24 \mathrm{~h}$ urine protein and urine red blood cell count were significantly lower than the control group $(P<0.05$ or $P<0.01)$.Two groups of patients before and after treatment of blood, routine, liver, kidney function,ECG, no abnormal changes. Conclusion: Tonifying kidney and spleen, detoxification of throat Chinese medicine can effectively improve patients with IgA nephropathy clinical symptoms, reduce $24 \mathrm{~h}$ proteinuria and urinary red blood cell count, and is safe and effective.
\end{abstract}

\section{Introduction}

$\operatorname{Ig}$ A nephropathy is referred to the $\operatorname{IgA}$ or $\operatorname{IgA}$ immune globulin in glomerular mesangial and capillary loop deposition caused by a series of clinical and pathological changes ${ }^{[1]}$. Its clinical manifestations are gross or microscopic hematuria, which may be associated with different levels of proteinuria, hypertension and impaired renal function.Studies have shown that IgA nephropathy usually due to infection or exacerbation of upper respiratory tract induced, which accounted for $80.1 \%$ of tonsillitis ${ }^{[2]}$. We believe that the two deficiency of spleen and kidney is the basis of the pathogenesis of IgA nephropathy.The main cause of treatment to cure the pharynx, use tonifying kidney and spleen, detoxification of throat decoction treatment ${ }^{[3]}$. We observed the use of tonifying kidney and spleen, detoxification of throat method in the treatment of 29 cases of IgA nephropathy are summarized as follows. 


\section{Clinical Data}

\section{Diagnosis and Dialectical Criteria}

Western medicine diagnosis standard is according to $<$ Nephrology $>$ [4] "diagnostic criteria of IgA nephropathy diseases". According to the standards of TCM "the guiding principle of clinical research on new drugs (Trial) "[5] and "Bian stone set (third Series) "[6]syndrome of spleen and kidney two empty, poison and blood stas is syndrome of pharynx. The main symptoms: 1) lumbar pain; 2) sore throat, throat swelling, including nuclear red, or even yellow white pus rot; 3) edema;4) anorexia and abdominal swelling; 5) fatigue. Secondary symptoms: 1) stool; 2) frequency urination; 3) dry mouth. Pale red tongue, scalloped, thin white fur, pulse weak. The main symptoms include 3 main symptoms,or1), 2) main symptoms added 2 items of secondary symptoms, tongue and pulse diagnosis can be es sential.

\section{Inclusion Criteria}

IgA nephropathy with western medicine and TCM diagnostic criteria standard; $24 \mathrm{~h}$ urine protein $<1 \mathrm{~g} ; 18$ to 65 years of age; informed consent.

\section{Exclusion Criteria}

A variety of secondary glomerulonephritis; with severe heart, lung and liver dysfunction; acute and chronic infectious diseases (including hepatitis, tuberculosis); acute nephritic syndrome, acute and chronic renal failure; period of pregnancy or breast-feeding women; a history of allergies.

\section{General Information}

From April 2012 to December 2014 in the Affiliated Hospital of Changchun University of Traditional Chinese Medicine Department of 60 nephropathy outpatient and hospitalized patients with $\operatorname{IgA}$ nephropathy were randomly divided into treatment group and control group, each of 30 cases. In the treatment group, 14 cases male, 16 female; the course of disease was 1 months to 4 years, the average $(7.34 \pm 14.44)$ months; age ranged from 18 to 62 years old, the average $(35.58 \pm 10.70)$ years old. In the control group, male 11 cases, female 19 cases; the duration ranged from 1 weeks to 3 years, the average $(6.78 \pm 10.83)$ months; age ranged from 19 to 61 years old, the average $(36.13 \pm 10.45)$ years old. No statistical significance between the two groups of patients with general information difference $(P>0.05)$ comparable.

\section{The Method}

\section{Treatment Method}

The treatment group was given tonifying kidney and spleen, detoxification of throat of Chinese herbal medicine orally.Medicine composition: Rehmannia 20g, Astragalus $20 \mathrm{~g}$, Dang shen $15 \mathrm{~g}$,Jin qiao mai $30 \mathrm{~g}$,Zi jin pi $30 \mathrm{~g}$, Wood butterfly $15 \mathrm{~g}$, turmeric $10 \mathrm{~g}$, Poria 50g,cogongrass rhizome $100 \mathrm{~g}$, Pu huang $15 \mathrm{~g}$, Calvatia $15 \mathrm{~g}$,Jin ying zi $20 \mathrm{~g}$. One. dose per day,by the manufacturing laboratory of the Affiliated Hospital of Changchun University of Traditional Chinese Medicine unified decoction, orally two times a day, a course of 3 months. The control group was treated with Benazepri Hydrochoride Tablets (Lotensin, per 10mg, Beijing Novartis Pharma Ltd, Zhunzi 1050423 H0) 10mg, orally 1 times a day; if there is hypertension, combined with the use of Levamlodipine Besylate Tablets (Shi Huida, 2.5 mg per tablet, Jilin Tianfeng Pharmaceutical Co. Ltd, Zhunzi H19991083) 2.5mg, orally 1 times a day a course of treatment 3 months. 


\section{Observation Index and Method}

Observation of the two groups before and after treatment in patients with TCM syndrome integral [5], $24 \mathrm{~h}$ urine protein, urine red blood cell count, and blood routine, urine routine and hepatorenal function change, ECG monitoring.

\section{Statistical Methods}

Using SPSS 19 statistical software, measurement data was descripted with $\left(\bar{x}_{ \pm} \mathrm{s}\right)$, paired-samples t-test was used. $P<0.05$ differences have statistical significance.

\section{Results}

Treatment group 1 case lost because He cannot tolerate the decoction of Chinese medicine taste; control group 3 cases lost, 1 case intolerant hypotension after Lotensin oral, and 2 cases intolerant dry cough after Lotensin oral.

\section{Comparison of TCM Syndromes before and after Treatment in Two Groups of Patients}

Table 1 showed the treatment group of lumbar pain, sore throat, fatigue, edema, low sodium, dry mouth and integral significantly decreased compared with those before treatment $(P<0.05)$; before and after treatment control group the integral of TCM syndrome had no significant changes $(P>0.05)$; comparison of the two groups after treatment showed the differences of scores were statistically significant $(P<0.05)$. The treatment group was lower than that in control group.

Notes:compare with before treatment of treatment group, ${ }^{*} P<0.05$; compare with after treatment of control group, ${ }^{\triangle} P<0.05$;

Table 1:Comparis on of TCM syndromes before and after treatment in two groups $(\mathrm{x} \pm \mathrm{S})$

\begin{tabular}{|c|c|c|c|c|c|c|c|c|}
\hline \multicolumn{3}{|c|}{ treame } & \multirow[b]{2}{*}{ Lumbar pain } & \multirow[b]{2}{*}{ Sore throat } & \multirow[b]{2}{*}{ Fatigue } & \multirow[b]{2}{*}{ Edema } & \multirow[b]{2}{*}{ Less satisfied } & \multirow[b]{2}{*}{ Dry mouth } \\
\hline group & nt & $\mathrm{N}$ & & & & & & \\
\hline $\begin{array}{c}\text { treatm } \\
\text { ent }\end{array}$ & Before & 29 & $4.07 \pm 1.56$ & 3. $27 \pm 1.03$ & 3. $47 \pm 1.46$ & $3.37 \pm 1.16$ & 1. $58 \pm 0.61$ & $1.74 \pm 0.71$ \\
\hline group & After & 29 & $1.67 \pm 1.0^{* \Delta}$ & $1.20 \pm 0.65^{* \Delta}$ & $1.40 \pm 0.97^{* \Delta}$ & $1.78 \pm 0.98^{\star \Delta}$ & $0.96 \pm 0.37^{\star \Delta}$ & $0.93 \pm 0.46^{* \Delta}$ \\
\hline $\begin{array}{c}\text { contro } \\
1\end{array}$ & Before & 27 & $3.87 \pm 1.51$ & $3.47 \pm 1.37$ & $3.33 \pm 1.44$ & $3.31 \pm 1.14$ & $1.65 \pm 0.68$ & $1.72 \pm 0.62$ \\
\hline group & After & 27 & $3.07 \pm 1.44$ & 3. $00 \pm 1.26$ & 2. $87 \pm 1.28$ & $2.72 \pm 1.08$ & 1. $58 \pm 0.70$ & $1.57 \pm 0.51$ \\
\hline
\end{tabular}

\section{Comparison of $24 \mathrm{~h}$ Urine Protein and Red Blood Cell Count between Two Groups before and after Treatment}

Table 2 showed that after treatment $24 \mathrm{~h}$ urinary protein and urinary red blood cell count of the treatment group were significantly reduced $(P<0.01)$; control group after treatment only $24 \mathrm{~h}$ urinary protein decreased significantly $(P<0.05)$, the difference in urinary red blood cell count has no statistical significance $(P>0.05)$. Comparison of the two groups after treatment showed $24 \mathrm{~h}$ urine protein and urine red blood cell count of the treatment group were significantly lower than the control group $(P<0.05$ or $P<0.01)$. 
Table 2. Comparison of $24 \mathrm{~h}$ urine protein and red blood cell count before and after treatment in two groups of patients with $\operatorname{IgAN}$

\begin{tabular}{ccccc}
\hline group & time & number & $\begin{array}{c}\text { 24 h urinary protein } \\
\text { quantitation }\end{array}$ & Urine red blood cell count \\
\hline \multirow{2}{*}{ treatment group } & Before treatment & 29 & $0.98 \pm 0.32$ & $14.37 \pm 5.68$ \\
& After treatment & 29 & $0.36 \pm 0.16^{* \star \Delta}$ & $4.78 \pm 1.26^{* * \Delta \Delta}$ \\
\multirow{2}{*}{ control group } & Before treatment & 27 & $0.89 \pm 0.33$ & $13.37 \pm 4.78$ \\
& After treatment & 27 & $0.62 \pm 0.21^{*}$ & $11.52 \pm 3.12$ \\
\hline
\end{tabular}

Notes:compare with before treatment of treatment group, $\quad P<0.01$, compare with before treatment of control group $P<0.05$; compare with after treatment of control group, ${ }^{\Delta} P<0.05, \Delta \Delta P<0.01$.

\section{Security Detection}

Two groups of patients before and after treatment conducted the blood routine, stool routine, liver function, kidney function, electrocardiogram monitoring, and no abnormal change. After oral Lotensin of the control group, 1 cases of intolerance to hypotension, 2 cases of cough.

\section{Conclusion}

In traditional Chinese medicine, IgA nephropathy belongs to "hematuria" "edema" "consumptivedisease" category.In kidney disease, involving the lung and spleen. Peiqing Zhang believes that this empty sign is the main pathogenesis of the disease [7]; Zong-li Zhang think IgA nephropathy onset of polygenetic feeling caused by wind heat evil or damp heat[8]. Chinese medicine master Jixue Ren have unique insights for diagnosis and treatment of this disease. Application of laryngeal kidney related theories system describes the diagnosis and treatment of the disease, which believes that the toxin stagnation throat is causative factors of the pathogenesis of IgA nephropathy[9]. "lingshu" said "the kidney meridian of foot Shao yin, the straight from the renal tubular liver and diaphragm, into the lungs, through the throat, with the tongue." Visible throat is kidney lung collaterals, for access to the evils of the portal and toxin from the nose and mouth, throat, forming milk moth, from blood invasion in the kidney, Fuxie formation, long and to poison, cause the disease, persistent unhealed.

Modern research confirms that the IgA nephropathy and tonsillitis patients whose $\operatorname{IgA}$ content in tonsillar tissue increased.The tonsils of $\operatorname{IgA}$ and $\operatorname{IgA}$ nephropathy mesangial deposition of $\operatorname{IgA}$ were $\mathrm{J}$ chain positive poly type $\operatorname{IgA}[10]$. Hong-dong Huang [11] found that accompanied by tonsil infection of IgA nephropathy patients whose tonsillar CD4 +, CD25 + cells reduced and J chain IgA secretion increase is main factor leading to IgA nephropathy, indicating that the pharyngeal tonsil infection is IgA nephropathy predisposing factors.And the imbalance of the innate immune system is the basis of IgA nephropathy.

The research results showed that the traditional Chinese medicine of tonifying kidney and spleen, detoxification of throat not only can improve patients clinical symptoms, but also reduce the $24 \mathrm{~h}$ proteinuria and urinary red blood cell count. Analysis shows that it relate to regulating the immune function of patients with IgA nephropathy, and effectively controling infection of the upper respiratory tract.

\section{Acknowledge ment}

Jilin Province Administration of traditional Chinese medicine science and technology project (No. 2014-ZC51,2014-ZD7) 


\section{Reference}

[1] BARRATT J, FEEHALLY J. IgA nephropathy [J]. J Am Soc Nephrol,2005,16( 7) : 2088-2097.

[2] Xie Yuansheng, Chen Xiangmei. The pathogenesis and treatment of the amygdala and IgA (in Chinese). The kidney disease 2004,20(4) : 300-302.

[3] Zi-kun Wang, Shou- lin Zhang, Hong-bao Zhang, et al. Study on the treatment of IgA nephropathy from pharynx (in Chinese). Journal of Changchun University of Traditional Chinese Medicine. 2015,31 (1) : 80-81.

[4] Hai-yan Wang. Kidney disease (in Chinese). 3 ed. Beijing: People's Medical Publishing House, China. 2008: 1002-1008.

[5] Xiaoyu Zheng. Guiding principle of clinical research on new drugs of traditional Chinese medicine (for Trial Implementation) (M) (in Chinese). Beijing: Chinese medical science and Technology Press. 2002: 156-162.

[6] Yu Jing.Bian Stone set(in Chinese): third series M. Shanghai: Shanghai University of Traditional Chinese Medicine press. 2002: 304-308.

[7] Na Liu, Liyan Wang. Peiqing Zhang's experience in treatment of IgA nephropathy with tonsil abnormal(in Chinese). Shanghai traditional Chinese medicine journal. 2008,42(6) : 10-11.

[8] Li Zhang, Xingkun Zhang, Zongli Zhang. Professor Zhang Zongli's experience in treating $\operatorname{IgA}$ nephropathy[J] (in Chinese). Journal of Changchun University of Traditional Chinese Medicine. 2011,27( 2) : 191-192.

[9] Yanhua Liu, Xijie Ren, Jian Wang, et al. Ren Jixue Professor application of laryngeal kidney related theory diagnosis and treatment of chronic renal experience $[\mathrm{J}]$ (in Chinese). TCM magazine.

[10] Mei Zhou, Wenguang Zhou, Honglin Yin. The tonsil immunohistochemistry in patients with $\operatorname{IgA}$ nephropathy $[\mathrm{J}]$ (in Chinese). Journal of clinical observation of Department of ENT 2000, 14(12); 536- 537.

[11]Hongdong Huang, Youming Peng, Hong Liu, et al. Influence of inactivated strains of tonsil IgA nephropathy tonsillar CD4+CD25+ cells and chain (in Chinese). Journal of renal transplantation. 2007, 16(3):215- 221, 300 